\title{
La doble carga de la malnutrición y nuestra responsabilidad como profesionales dedicados a la nutrición
}

\author{
The double burden of malnutrition and our \\ responsibility as nutrition professionals \\ O duplo fardo da malnutrição e nossa responsabilidade \\ como profissionais dedicados à nutrição
}

Fanny Aldana-Parra, ND, PhD

https://doi.org/10.35454/rncm.v3n2.198

Los Objetivos de Desarrollo Sostenible marcaron un compromiso para las naciones en el logro de un mundo sin hambre, con seguridad alimentaria y sin malnutrición en cualquiera de sus formas para el año $2030^{(1)}$. Desafortunadamente, en 2018 se registró un aumento en la subalimentación en América Latina y el Caribe que asciende a 42,5 millones de personas y una cifra de inseguridad alimentaria de 187 millones de personas en nuestra región ${ }^{(2)}$. Esta subalimentación, que podría tener como una de sus causas la inseguridad alimentaria, sería uno de los factores determinantes tanto de la desnutrición como del sobrepeso y la obesidad ${ }^{(3)}$; este fenómeno se entiende como un consumo habitual e inadecuado de alimentos, lo cual conlleva a un suministro insuficiente de energía para el individuo, o un suministro insuficiente de micronutrientes como consecuencia de una dieta energéticamente mal balanceada ${ }^{(4)}$.

El reto es doble y enorme. Significa enfrentar la doble carga de la malnutrición tanto por déficit como por exceso con el agravante de la incertidumbre que trae la pandemia, probablemente con un aumento en las cifras de inseguridad alimentaria como consecuencia de la emergencia económica mundial y de las disrupciones en la cadena de suministro de alimentos que se han visto afectadas por el decrecimiento en las cosechas de alimentos, el cierre de plantas procesadoras de alimentos y el de fronteras entre países y ciudades. Se estima que la cifra de personas en estado de inseguridad alimentaria podría duplicarse a finales de 2020 como consecuencia de la pandemia que se vive en la actualidad y esto tendrá implicaciones graves en el estado nutricional, que posiblemente nos conducirá a una crisis nutricional, con un aumento en enfermedades crónicas no transmisibles.

En consecuencia, estamos ad portas de una emergencia alimentaria, definida como una situación extraordinaria en la cual las personas no son capaces de alcanzar sus necesidades básicas para sobrevivir ${ }^{(5)}$. Los profesionales de la salud dedicados a la nutrición somos actores fundamentales en la identificación del riesgo nutricional, prescripción de dietas saludables y balanceadas que sean parte de sistemas sostenibles de alimentos ${ }^{(6)} \mathrm{y}$ en el seguimiento del estado nutricional en todos los ámbitos poblacionales.

El valor que tiene el presente número de la Revista de Metabolismo y Nutrición Clínica radica no solo en los diferentes temas que abordan diversos tipos de dietas para patologías específicas, sino también en que fue realizado durante la pandemia y el confinamiento, momento en el que los autores hicieron su mayor esfuerzo por presentarles el mejor análisis de sus resultados y en que la editora y el comité editorial nunca dejaron de lado su objetivo de seguir adelante con este propósito que trajo además, la indexación de la Revista.

En 2050 seremos 10 billones de habitantes en nuestro planeta con muchos retos nutricionales por delante, seamos nosotros ejemplo de tenacidad, esa misma que nos han mostrado Diana Cárdenas y todo su equipo.

Fanny Aldana fue Editora Asociada de la Revista hasta marzo de 2020. Con entusiasmo y gran pertinencia ayudó a consolidar la revista en esta etapa previa a la indexación. Sus recomendaciones y su visión de la investigación fueron muy importantes para la revista. Las puertas siempre estarán abiertas para ti. ¡Gracias Fanny! 


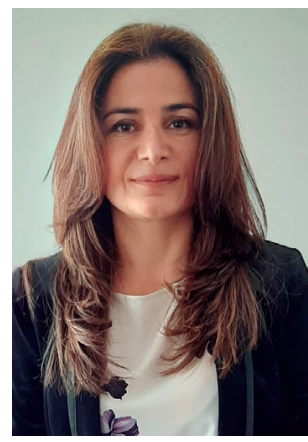

Fanny Aldana-Parra, ND, PhD.

Nutricionista-Dietista de la Pontificia Universidad Javeriana. Epidemióloga clínica de la Universidad El Bosque. Doctora en Ciencias Biológicas de la Pontificia Universidad Javeriana. Estancia Doctoral en el Institute of Child Health de University College London en Reino Unido. Pertenece al grupo de expertos temáticos de Colciencias-Ministerio de Salud en la elaboración de la Guía de Práctica Clínica para el diagnóstico y prevención de obesidad para la población adulta colombiana. Sus líneas de investigación están relacionadas con cirugía bariátrica y metabólica, factores de riesgo de obesidad en población colombiana y en estrategias eficientes en consejería nutricional. Es par evaluador de Clinical Nutrition ESPEN, PlosOne, Revista de la Facultad de Medicina de la Universidad Nacional y Revista Colombiana de Pediatría.

\section{Referencias bibliográficas}

1. Griggs D, Stafford-Smith M, Gaffney O, Rockström, Öhman MC, Shyamsundar P, et al. Sustainable development goals for people and planet. Nature. 2013; 495: 305-7. doi: https://doi. org/10.1038/495305a.

2. World Health Organization. World Health Statistics: monitoring health for the SDGs, sustainable development goals [Internet]. Geneva: World Health Organization; 2018 [Fecha de consulta: 1 de agosto 2020]. Disponible en: https://apps. who.int/iris/handle/10665/272596.

3. Wells JC, Sawaya AL, Wibaek R, Mwangome M, Poullas MS, Yajnik CS, et al. The double burden of malnutrition: aetiological pathways and consequences for health. Lancet. 2020;395(10217):75-88. https://doi.org/10.1016/S01406736(19)32472-9.
4. FAO, FIDA, OMS, PMA y UNICEF. El estado de la seguridad alimentaria y la nutrición en el mundo 2019. Protegerse frente a la desaceleración y el debilitamiento de la economía [Internet]. Roma: FAO; 2019 [Fecha de consulta: 1 de agosto 2020]. Disponible en: http://www.fao.org/3/ca5162es/ ca5162es.pdf

5. United Nations. Policy brief: The impact of COVID-19 on food security and nutrition [Internet]. June 2020 [Fecha de consulta: 1 de agosto 2020]. Disponible en: https://www. tralac.org/documents/resources/covid-19/3813-the-impactof-covid-19-on-food-security-and-nutrition-un-policy-briefjune-2020/file.html.

6. Willett W, Rockström J, Loken B, Springmann M, Lang T, Vermeulen S, et al. Food in the Anthropocene: the EATLancet Commission on healthy diets from sustainable food systems. Lancet. 2019;393(10170):447-92. doi: https://doi. org/10.1016/S0140-6736(18)31788-4. 